\title{
Worldwide Clinical Practice of High-Flow Nasal Cannula and Concomitant Aerosol Therapy in the Adult ICU Setting
}

\author{
Jie Li, Meilien Tu, Lei Yang, Guoqiang Jing, James B Fink, Chris Burtin, \\ Armèle Dornelas de Andrade, Lingyue Gong, Lixin Xie, and Stephan Ehrmann
}

\begin{abstract}
BACKGROUND: High-flow nasal cannula (HFNC) oxygen therapy has been broadly used. However, no consensus has been achieved on the practical implementation of HFNC and how to provide aerosol delivery during HFNC therapy in adult patients. METHODS: An online anonymous questionnaire survey endorsed by 4 academic societies from America, Europe, mainland China, and Taiwan was administered from May to December 2019. Clinicians who had worked in adult ICUs for $>1$ year and had used HFNC to treat patients within 30 days were included. RESULTS: A total of 2,279 participants clicked on the survey link, 1,358 respondents completed the HFNC section of the questionnaire, whereas 1,014 completed the whole survey. Postextubation hypoxemia and moderate hypoxemia were major indications for HFNC. The initial flow was mainly set at 40-50 $\mathrm{L} / \mathrm{min}$. Aerosol delivery via HFNC was used by $24 \%$ of the participants $(248 / 1,014), 30 \%(74 / 248)$ of whom reported reducing flow during aerosol delivery. For the patients who required aerosol treatment during HFNC therapy, $40 \%$ of the participants $(403 /$ 1,014) reported placing a nebulizer with a mask or mouthpiece while pursuing HFNC whereas $33 \%(331 / 1,014)$ discontinued HFNC to use conventional aerosol devices. A vibrating mesh nebulizer was the most commonly used nebulizer $(40 \%)$ and was mainly placed at the inlet of the humidifier. CONCLUSIONS: The clinical utilization of HFNC was variable, as were indications, flow settings, and criteria for adjustment. Many practices associated with concomitant aerosol therapy were not consistent with available evidence for optimal use. More efforts are warranted to close the knowledge gap. Key words: High-flow nasal cannula; aerosol therapy; survey; hypoxemia; transnasal pulmonary aerosol delivery. [Respir Care 2021;66(9):1416-1424. (C) 2021 Daedalus Enterprises]
\end{abstract}

\section{Introduction}

High-flow nasal cannula (HFNC) is a relatively new modality of oxygen therapy that delivers heated and humidified oxygen-enriched gas at high flows potentially exceeding the patient's inspiratory flow demand. ${ }^{1,2}$ Multiple randomized controlled trials and meta-analyses have demonstrated the superiority of HFNC over conventional oxygen and the noninferiority to noninvasive ventilation as a method to improve oxygenation and ultimately avoid intubation or re-intubation in subjects who are hypoxemic. ${ }^{3-10}$ Increasing evidence has supported the use of HFNC to reduce the work of breathing, improve ventilation, and alleviate hypercapnia among patients with COPD. ${ }^{11-15}$

Flow setting plays a critical role in HFNC utilization because an increased flow can reduce inspiratory effort and improve ventilation and dynamic lung compliance. ${ }^{16,17}$ Carbon dioxide has been shown to decrease as the dead space in the upper airway is washed out by the high gas flow. ${ }^{18}$ However, criteria for the flow setting have not yet achieved consensus in clinical trials and have varied from 10-15 L/min to $50-60 \mathrm{~L} / \mathrm{min}$ (see the supplementary materials at http:// www.rcjournal.com), even in the same population (eg, postoperative extubation). ${ }^{19}$ Surveys from pediatric clinicians observed that the utilization of HFNC, including flow initiation and titration, patient assessment, and HFNC weaning varied greatly. ${ }^{20,21}$ A French nationwide survey of ICU physicians reported that the daily practice of HFNC, such as the criteria for HFNC initiation, weaning, and failure, was heterogeneous. ${ }^{22}$ The practice in other countries and areas has not been reported. To our knowledge, no clinical guideline has been established on how to apply HFNC. 


\section{Worldwide Clinical Practice of HFNC}

Moreover, aerosol delivery via HFNC has attracted clinicians' interest in recent years due to its combined benefits from HFNC and aerosolized medication. ${ }^{23} \mathrm{~A}$ survey among American pediatric respiratory therapists showed that $75 \%$ of them used HFNC to deliver aerosol therapy for pediatric patients. ${ }^{20}$ However, the utilization of aerosol therapy methods for adult patients during HFNC therapy is still unknown. Thus, to understand the current clinical practice, we implemented a worldwide survey on the utilization of HFNC among ICU clinicians who worked with adult patients, with some focus on the concomitant delivery of aerosol therapy.

\section{Methods}

After a thorough literature review and discussion with 6 academic respiratory care experts with $\geq 10$ years of ICU experience and university faculty positions, we designed a questionnaire by using an online survey platform (SurveyMonkey, San Mateo, California). This questionnaire included 2 main sections: the technical application of HFNC in an ICU and aerosol delivery during HFNC therapy. De-identified demographic information was also collected (the questionnaire is available in the supplementary materials at http://www.rcjournal.com). The questionnaire was sent to 10 clinicians who worked at the bedside and used HFNC for $\geq 5$ patients/mo and feedback was integrated. This study, according to the local requirement, was approved by 4 major ethical committees: Rush University, Chicago, Illinois (18121305-IRB01-AM01), French Intensive Care Society, Paris, France (CE SRLF 19-18), Chang Gung Medical Foundation, Taiwan (201900250B0), and People's Liberation Army General

\section{QUICK LOOK}

\section{Current knowledge}

High-flow nasal cannula (HFNC) has been broadly used to improve oxygenation and avoid intubation and/or re-intubation for patients who are hypoxemic. More recently, HFNC has been extended to other patient populations, such as patients with COPD, to assist endoscopy examination, intubation, and in other clinical scenarios.

\section{What this paper contributes to our knowledge}

This worldwide survey revealed a wide range of practices, which suggests knowledge gaps between the current practice and evidence. Clinical guidance on HFNC administration and concomitant aerosol therapy are urgently needed; educational efforts or quality improvement projects are warranted to close the knowledge gap.

Hospital, Beijing, China (S2019-047-01). The need for informed consent was waived.

The survey was conducted from May 24 to December 31, 2019. The study was endorsed by the American Association for Respiratory Care, the European Society of Intensive Care Medicine, the Chinese Respiratory Disease Society, and the Taiwan Respiratory Care Society. The survey's invitation was posted on their social media platforms, for example, web page, Facebook, and Twitter. Respondents who fulfilled all of the following 3 criteria were enrolled in the study: (1) provided care for adult patients in the ICU, (2) had $>1$ year of work experience in an ICU, and (3) used HFNC for patients in the past 30 days.

Dr Li declares relationships with Fisher \& Paykel Healthcare, Aerogen, the Rice Foundation, the American Association for Respiratory Care; she also serves as Section Editor for Respiratory CARE. Dr Fink discloses relationships with Aerogen Pharma. Dr Ehrmann discloses relationships Aerogen, Fisher \& Paykel Healthcare, and Hamilton Medical. The other authors have no conflicts to disclose.

Supplementary material related to this paper is available at http://www. rcjournal.com

Correspondence: Jie Li PhD RRT RRT-ACCS RRT-NPS FAARC, Division of Respiratory Care, Department of Cardiopulmonary Sciences, Rush University, 600 S Paulina St, Suite 765, Chicago, IL. 60612. Email: Jie_Li@rush.edu or Lixin Xie MD, Department of Respiratory and Critical Care Medicine, People's Liberation Army General Hospital, 28 Fuxing Road (Wukesong), Beijing, China. E-mail: xielx301@126.com.

DOI: $10.4187 /$ respcare.08996 


\section{Worldwide Clinical Practice of HFNC}

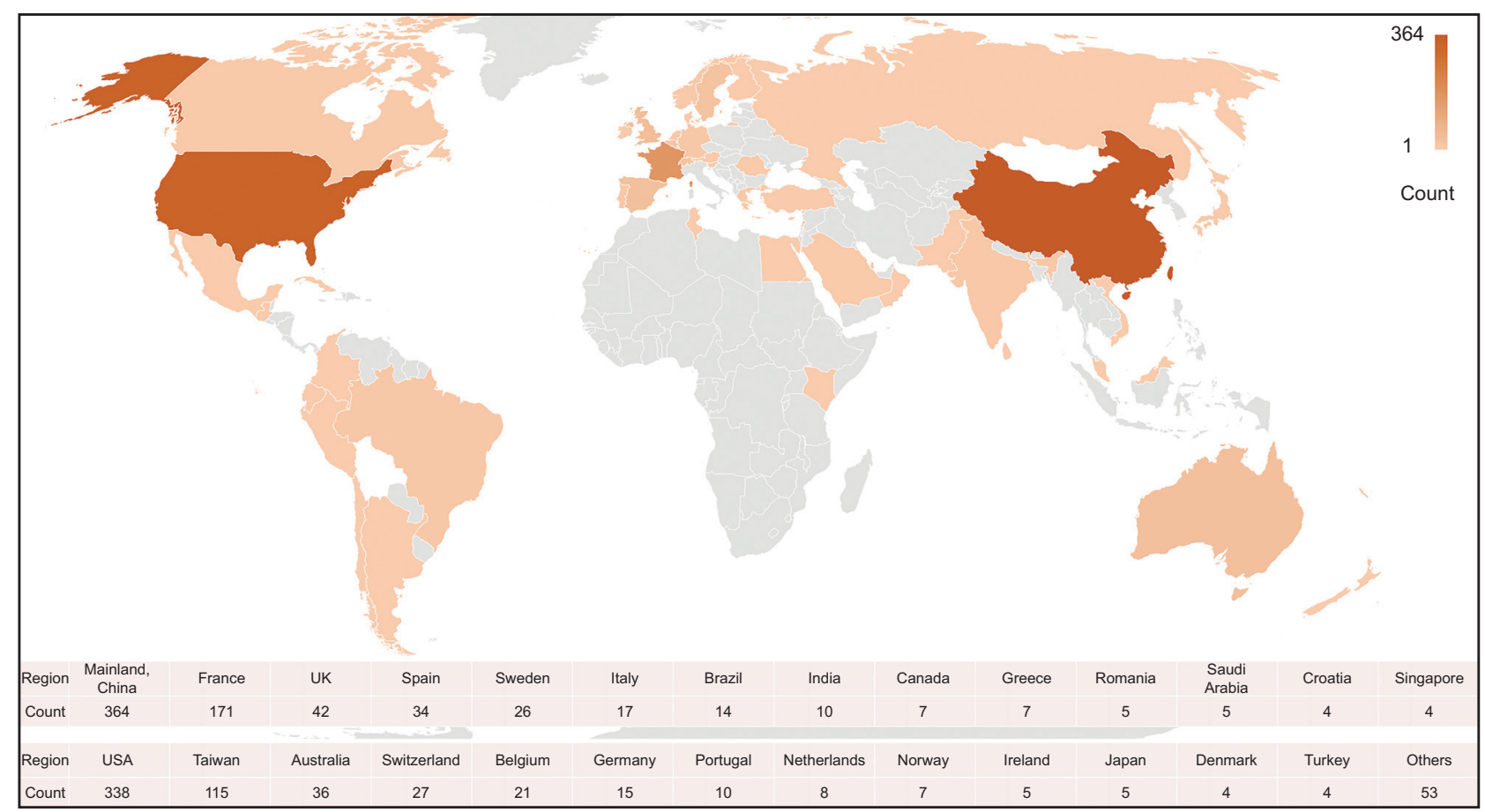

Fig. 1. Global distribution of the 1,358 participants. Among these 1,358 participants, the majority were from mainland China (27\%); Taiwan (8\%); United States (25\%); and Europe (30\%), including France, United Kingdom, Spain and others.

\section{Data Analysis}

Questionnaires with incomplete responses, defined as $>10 \%$ of questions in the first section of the questionnaire left unanswered, were not analyzed. Continuous variables were reported as mean $\pm \mathrm{SD}$ or median (interquartile range [IQR]), whereas categorical variables were reported as frequency and proportion. Two independent investigators (GJ and LG) reviewed the answers to open questions. The chi-square test or the Fisher exact test was used for comparing categorical variables. All the analyses were performed with SPSS 26.0 (SPSS, Chicago, Illinois), and a 2 -sided $P$ value of $<.05$ was considered statistically significant.

\section{Results}

Among the 2,279 respondents who clicked on the survey link, 755 were excluded because (1) 242 were not working in an adult ICU, (2) 114 worked in an adult ICU for $<1$ year, (3) 362 had not used HFNC to treat patients in the past 30 days, and (4) 37 had previously filled out the survey. Among the remaining 1,524 participants, 166 were excluded because they did not complete the first section of the questionnaire. A total of 1,358 respondents from 61 countries and areas completed the HFNC section (Fig. 1); of them, 1,014 continued to complete the aerosol therapy section but only 988 offered their demographic information. Among these 988 participants, 188 (19\%) were from North America and 92\% were respiratory therapists; 428 (43\%) were from mainland China and Taiwan and $48 \%$ and $32 \%$ were nurses and respiratory therapists, respectively; $372(38 \%)$ were from Europe and other areas and $76 \%$ were physicians. Overall, $72 \%$ worked in academic institutions, with a mean \pm SD $12.2 \pm 9.2$ years of ICU working experience, $53 \%$ (521/977) worked in leadership position, including as director, attending physician, manager, educator, and supervisor (Table 1).

\section{Utilization of HFNC}

The questions of this section were answered by 1,358 participants. According to them, 25\% of participants' institutions had a HFNC protocol, whereas others administrated HFNC empirically.

HFNC Indications. Overall, the top 4 indications for HFNC were the following: postextubation hypoxemia (78\%), moderate hypoxemia, with $\mathrm{P}_{\mathrm{aO}} / \mathrm{F}_{\mathrm{IO}_{2}}$ at $100-200$ $\mathrm{mm} \mathrm{Hg}(73 \%)$; mild hypoxemia, with $\mathrm{P}_{\mathrm{aO}_{2}} / \mathrm{F}_{\mathrm{IO}_{2}}$ at 201-300 $\mathrm{mm} \mathrm{Hg} \mathrm{(56 \% );} \mathrm{and} \mathrm{postextubation} \mathrm{of} \mathrm{COPD} \mathrm{(51 \% )} \mathrm{(Fig.}$ 2). The former 2 indications were common among all the participants; however, HFNC to improve or maintain 


\section{Worldwide Clinical Practice of HFNC}

Table 1. Participants' Demographic Information

\begin{tabular}{|c|c|c|c|c|}
\hline Demographics & $\begin{array}{c}\text { Overall } \\
(n=988)\end{array}$ & $\begin{array}{l}\text { North America } \\
\quad(n=188)\end{array}$ & $\begin{array}{l}\text { Mainland China and Taiwan } \\
\qquad(n=428)\end{array}$ & $\begin{array}{c}\text { Europe and Other areas } \\
\qquad(n=372)\end{array}$ \\
\hline \multicolumn{5}{|l|}{ Profession, $n(\%)(n=988)$} \\
\hline MDs & 375 (38) & $13(7)$ & $80(19)$ & $282(76)$ \\
\hline RTs & $323(33)$ & $173(92)$ & $138(32)$ & $12(3)$ \\
\hline RNs & $224(23)$ & $2(1)$ & $204(48)$ & $18(5)$ \\
\hline PTs & $60(6)$ & 0 & $2(0)$ & $58(16)$ \\
\hline Others & $6(1)$ & $0(0)$ & $4(1)$ & $2(1)$ \\
\hline \multicolumn{5}{|l|}{ Hospital, $n(\%)(n=984)$} \\
\hline Community & $209(21)$ & $87(47)$ & $10(2)$ & $112(30)$ \\
\hline Academic & $713(72)$ & $100(53)$ & $356(83)$ & $257(70)$ \\
\hline Others & $62(6)$ & 0 & $62(14)$ & 0 \\
\hline \multicolumn{5}{|l|}{ Job title, $n(\%)(n=977)$} \\
\hline Attending MD, or RN, RT, or PT director or manager & $280(29)$ & $47(25)$ & $58(14)$ & $175(48)$ \\
\hline $\mathrm{RT}$ or $\mathrm{RN}$ educator & $110(11)$ & $17(9)$ & $90(21)$ & $3(1)$ \\
\hline Fellow MD, or RT or PT supervisor & $131(13)$ & $28(15)$ & $63(15)$ & $40(11)$ \\
\hline Resident, or RN, RT, or PT staff & $394(40)$ & $92(49)$ & $199(47)$ & $103(28)$ \\
\hline Others & $62(6)$ & $2(1)$ & $15(4)$ & $45(12)$ \\
\hline \multicolumn{5}{|l|}{ Degree, $n(\%)(n=978)$} \\
\hline Associate & $116(12)$ & $66(35)$ & $31(7)$ & $19(16)$ \\
\hline Bachelor & $442(45)$ & $86(46)$ & $313(73)$ & $43(10)$ \\
\hline Master & $178(18)$ & $23(12)$ & $65(15)$ & $90(51)$ \\
\hline Doctorate & $231(24)$ & $11(6)$ & $16(4)$ & $204(88)$ \\
\hline Others & $11(1)$ & 0 & $3(1)$ & $8(73)$ \\
\hline ICU working experience, mean \pm SD y $(n=965)$ & $12.2 \pm 9.2$ & $19.2 \pm 11.5$ & $8.6 \pm 5.6$ & $13.3 \pm 9.0$ \\
\hline \multicolumn{5}{|l|}{$\begin{array}{l}\mathrm{MD}=\text { medical doctor } \\
\mathrm{RT}=\text { respiratory therapist } \\
\mathrm{RN}=\text { registered nurse } \\
\mathrm{PT}=\text { physical therapist }\end{array}$} \\
\hline
\end{tabular}

oxygenation peri-intubation was more commonly used in Europe than in all other areas $(53 \%$ vs $38 \% ; P<.001)$, whereas HFNC for postextubation of COPD was more commonly used in China than in other areas (66\% vs $43 \%$; $P<.001)$.

Flow and Temperature Settings. Of the participants, 59\% set the same initial flow for all adult patients, with 29, 25, and $20 \%$ of the participants having set flows at 40,50 , and $60 \mathrm{~L} / \mathrm{min}$, respectively; whereas the remaining participants stated that they set initial flow according to the patients' diseases, and $40(35,50) \mathrm{L} / \mathrm{min}$ was the most common flow setting. HFNC flow was adjusted primarily based on patients' comfort $(81 \%)$, oxygenation $(77 \%)$, and breathing pattern $(70 \%)$. Nine percent of the participants deemed that the HFNC flow was set to match the patient's inspiratory flow, 36\% set the flow just exceeding the patient's inspiratory flow, whereas $28 \%$ set the flow much higher than the patient's inspiratory flow. However, none of them reported measuring the patient's inspiratory flow before initiation. By their estimation, the inspiratory flows for patients with mild, moderate, and severe hypoxemia were $35(25,40)$, $40(30,50)$, and $50(30,60) \mathrm{L} / \mathrm{min}$, respectively; whereas the inspiratory flows for subjects with stable COPD and COPD exacerbation were $30(20,40)$ and $40(30,55)$ $\mathrm{L} / \mathrm{min}$, respectively. With regard to temperature settings during HFNC treatment, $52 \%$ of the participants set it at $37^{\circ} \mathrm{C}$, whereas $30 \%$ and $14 \%$ set it at $34^{\circ} \mathrm{C}$ and $31^{\circ} \mathrm{C}$, respectively.

HFNC Weaning. When weaning from HFNC was considered, $52 \%$ and $13 \%$ of the participants preferred to first reduce $\mathrm{F}_{\mathrm{IO}_{2}}$ versus to first reduce the flow, respectively; whereas $25 \%$ decreased flow and $\mathrm{F}_{\mathrm{IO}_{2}}$ alternately.

HFNC Events. Nasal cannula dislodgement and rainout were the top 2 concerns during the utilization of HFNC. The incidence of ear or face skin breakdown $>6$ patients/y was also reported by $11 \%$ of the participants.

\section{Aerosol Delivery via HFNC}

The questions of this section were answered by 1,014 participants. When patients required aerosol treatment during HFNC therapy, 403 of the participants (40\%) placed the nebulizer via a mask or mouthpiece while pursuing HFNC therapy with the cannula in place, 331 (33\%) 


\section{Worldwide Clinical Practice of HFNC}

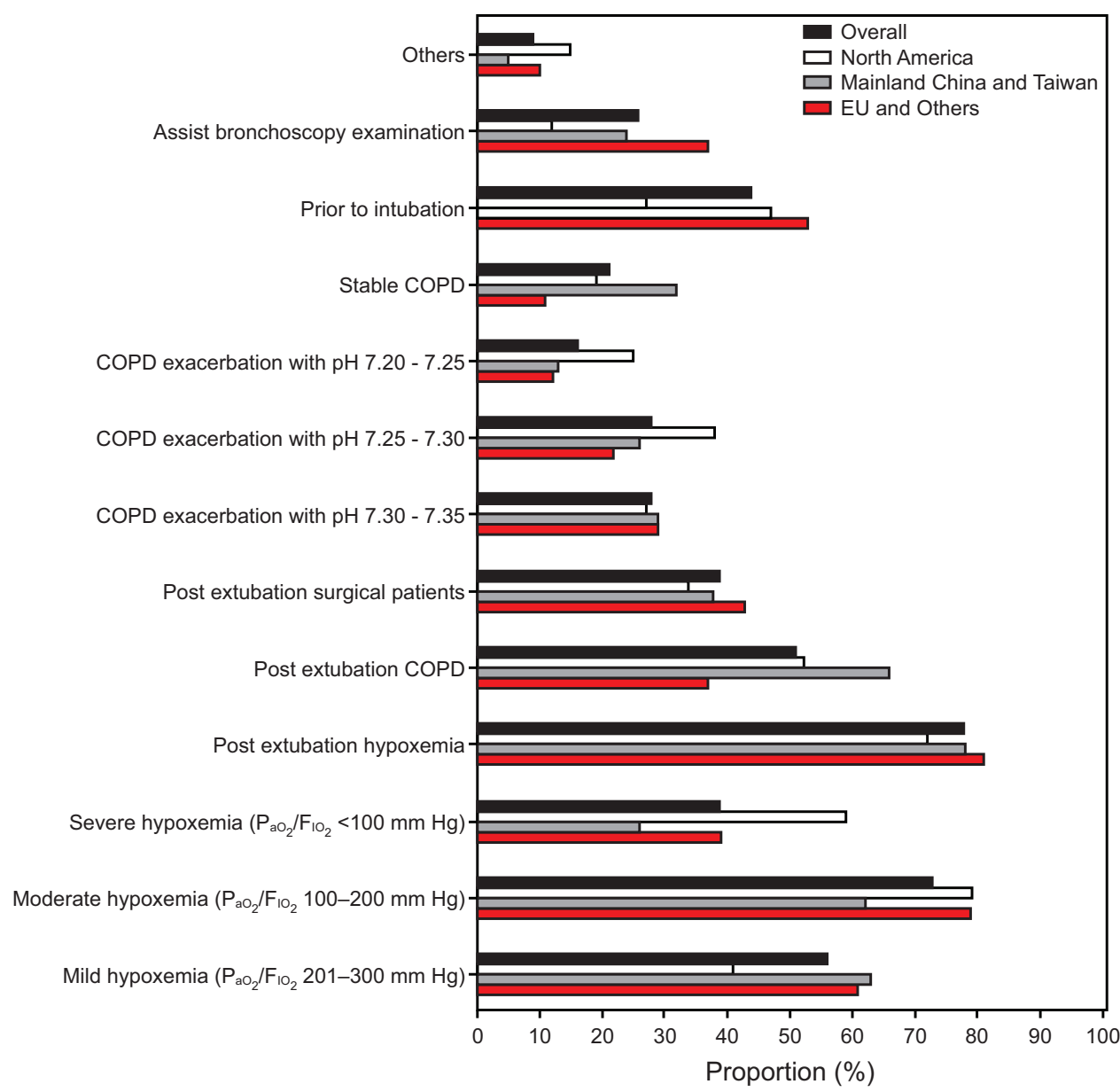

Fig. 2. High-flow nasal cannula (HFNC) indications. Overall (red bar), postextubation hypoxemia was the top HFNC indication, followed by moderate hypoxemia, mild hypoxemia and postextubation COPD. In North America, more clinicians preferred to use HFNC to treat severe hypoxemia than other areas; whereas, in China, more clinicians used HFNC to facilitate extubation for patients with COPD, and more European clinicians used HFNC to improve or maintain peri-intubation oxygenation.

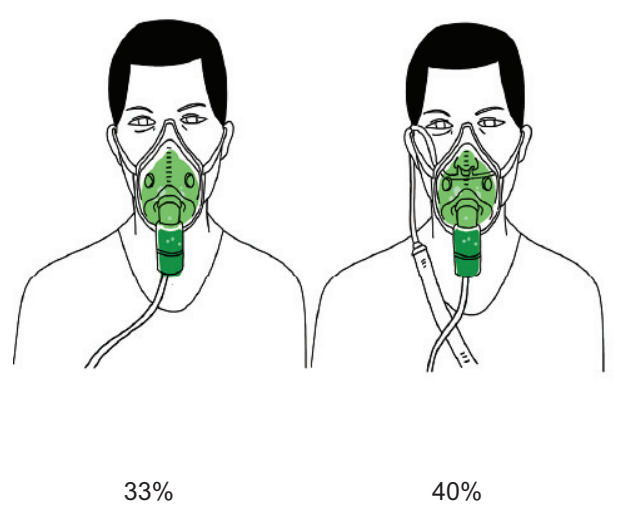

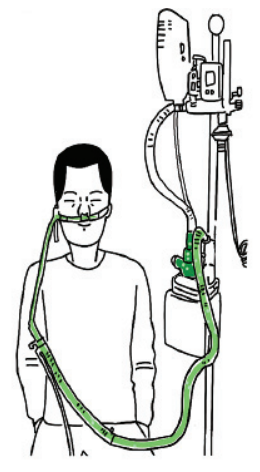

$24 \%$
Vibrating mesh nebulizer

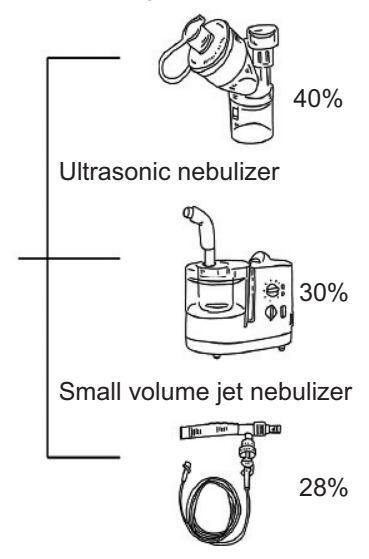

Fig. 3. Aerosol therapy during high-flow nasal cannula (HFNC) treatment. When patients required short-term aerosol treatment during HFNC therapy, $33 \%$ of the participants $(331 / 1,014)$ discontinued HFNC treatment to use a conventional aerosol device, $40 \%(403 / 1,014)$ placed the nebulizer with a mask over the nasal cannula, and $24 \%(248 / 1,014)$ placed the nebulizer in-line within the HFNC circuit. Of the 248 participants who delivered aerosol via HFNC, $40 \%$ used a vibrating mesh nebulizer, $30 \%$ used an ultrasonic nebulizer, and $28 \%$ used a small-volume jet nebulizer. 


\section{Worldwide Clinical Practice of HFNC}

discontinued HFNC treatment to use a conventional aerosol device, and 248 (24\%) placed the nebulizer in-line within the HFNC circuit (Fig. 3). For the 248 participants who administered aerosols this way, the use of a vibrating mesh nebulizer, ultrasonic nebulizer, and small-volume jet nebulizer were 40, 30, and 28\%, respectively (Fig. 3). Aerosol delivery via HFNC was more common in North America than in all other areas (44\% vs $20 \% ; P<.001$ ), and the vibrating mesh nebulizer was the preferred nebulizer (75\% [60/80]) in North America. The vibrating mesh nebulizer was primarily placed at the inlet of the humidifier (57\%), whereas the small-volume jet nebulizer was primarily placed close to the patient $(58 \%)$.

Among the 248 participants, 74 (30\%) reduced flow during aerosol therapy to increase aerosol deposition (68\%) and to improve patient's comfort (34\%), 27, 26, and $34 \%$ of these 74 participants reported to reduce flow to 10,20 , and $30 \mathrm{~L} / \mathrm{min}$, respectively. The top 3 medications that were frequently delivered $(>12$ patients/y) via HFNC were albuterol (68\%), ipratropium (66\%), and budesonide $(40 \%)$. Compared with conventional aerosol therapy, 177 of 248 of the participants (71\%) preferred to place the nebulizer in-line within the HFNC circuit for the purpose of maintaining HFNC benefits; 50, 49, and $40 \%$ of the participants claimed it was for better comfort, more efficiency, and more convenience, respectively.

Forty-five percent of the participants never used continuous nebulization; $20 \%$ of the participants reported in-line placement of a nebulizer via HFNC to deliver continuous aerosol, especially in North America (38\%); whereas $23 \%$ placed a mask with the nebulizer on top of HFNC, and 9\% discontinued HFNC to use conventional nebulizer continuously. Among the 766 participants who did not provide aerosol therapy via HFNC, 54\% had never heard of this delivery route and $27 \%$ reported a lack of evidence to support its utilization, whereas $17 \%$ deemed that the aerosol deposition in the lung was low.

\section{Discussion}

To our knowledge, this was the first worldwide survey on the utilization of HFNC and aerosol delivery during HFNC therapy in adult populations. HFNC was primarily used for patients who were hypoxemic, specifically for those with moderate hypoxemia and postextubation hypoxemia. HFNC was mainly implemented based on clinicians' experience, with only one fourth of the participants' institutions having established a protocol. The participants agreed that HFNC flow should be set higher than the patient's inspiratory flow, which was estimated to be variable based on the patient's disease. One fourth of the participants reported aerosol delivery via HFNC. For those participants who did not use an in-line nebulizer with HFNC, more than half of them placed a mask or mouthpiece with the nebulizer on top of the nasal cannula, whereas the remaining participants discontinued HFNC to deliver conventional aerosol therapy.

\section{HFNC Administrations}

Predominant evidence has been established for patients with acute hypoxemic respiratory failure to avoid intubation $^{3,10}$ and for postextubation ${ }^{4,7,8}$ and postoperative patients ${ }^{5,6}$ to reduce postextubation respiratory failure or re-intubation compared with conventional oxygen therapy. A recent metaanalysis demonstrated that the use of HFNC for preoxygenation before intubation of adult subjects who are hypoxemic reduced the risk of intubation-related complications compared with conventional oxygen therapy, but subjects treated with HFNC still had a higher incidence of desaturation than with noninvasive ventilation. ${ }^{9}$

The indications for HFNC in the present survey were aligned with the current evidence, except for the use of HFNC for preoxygenation before intubation, only $44 \%$ of the participants considered it as an HFNC indication, especially in North America, where only $27 \%$ of the clinicians used HFNC for preoxygenation, compared with 53\% in Europe. This difference might be due to the familiarity of this pre-oxygenation modality and the clinical urgency of intubation. So far, 6 of 7 randomized controlled trials that used HFNC peri-intubation were completed in France, ${ }^{9}$ which may explain the high percentage of French ICU physicians (84\%) who agreed with the use of HFNC for pre-intubation oxygenation. ${ }^{22}$

Interestingly, the participants preferred to use HFNC for patients with moderate hypoxemia versus those with mild or severe hypoxemia ( $73 \%$ vs $56 \%$ or $39 \%$ ). However, Shen et $\mathrm{al}^{24}$ performed a subgroup analysis in all the HFNC randomized controlled trials and found that the trials with $\mathrm{P}_{\mathrm{aO}_{2}} / \mathrm{F}_{\mathrm{IO}_{2}}>200 \mathrm{~mm} \mathrm{Hg}$ had greater benefits than trials with $\mathrm{P}_{\mathrm{aO}_{2}} / \mathrm{F}_{\mathrm{IO}_{2}} \leq 200 \mathrm{~mm} \mathrm{Hg}$, specifically for subjects after extubation. Future studies are needed to clarify the role of HFNC in patients with different severities and pathophysiologies. Despite the insufficient evidence supporting HFNC for patients with hypercapnia, one fourth of the participants used HFNC for patients with COPD, whether stable or during exacerbation.

When the HFNC flow is set to match or exceed the patient's inspiratory flow, $\mathrm{F}_{\mathrm{IO}_{2}}$ begins to be stabilized and an incremental increase of PEEP occurs linearly, with an increase in HFNC flow. ${ }^{25,26}$ Most investigators suggest that the HFNC flow should be set higher than or equal to a patient's inspiratory flow. One should attempt to reach the inspiratory flow of the patient, not be lower, and should eventually exceed it to have some safety because 


\section{Worldwide Clinical Practice of HFNC}

inspiratory flow may be variable from one breath to another. Because inspiratory flow is not commonly measured at the bedside, flow is commonly chosen based on an observed response to changes in oxygenation. A patient's peak inspiratory flows can vary from 20 to $100 \mathrm{~L} / \mathrm{min}$; in some cases, it may not be possible to meet the patient's peak inspiratory flow. Future studies are required to validate if the estimated flow by the participants correlated to the actual inspiratory flow of patients. Furthermore, from the summarization of the flow settings and adjustment in all the randomized controlled trials (see the supplementary materials at http://www.rcjournal.com), the flow settings varied in different diseases and countries; this was in agreement with our finding that more than three fourths of the participants' institutions had not established an HFNC protocol.

\section{Aerosol Delivery During HFNC Therapy}

One fourth of the participants reported placing a nebulizer in-line within the HFNC circuit to deliver aerosol, especially in North America; however, its utilization was lower than in a previous survey among American pediatric respiratory therapists $(47 \%$ vs $75 \%),{ }^{20}$ which might be explained by the difference in tolerance issue between the adult and pediatric populations with conventional aerosol devices, such as a small-volume jet nebulizer with face masks or mouthpieces. Cold aerosol, mask on the face, and noise from the nebulizer have been reported to irritate small children, which results in little to no inhaled dose. ${ }^{27}$ This population was observed to better tolerate in-line placement of nebulizer with HFNC. ${ }^{28-30}$ In adult subjects with stable asthma or COPD, 3 clinical trials reported similar efficacy of inhaling a bronchodilator via $\mathrm{HFNC}$ at $15-35 \mathrm{~L} / \mathrm{min}$ versus a conventional aerosol device. ${ }^{31-33}$

Likewise, for subjects with pulmonary hypertension and/or refractory hypoxemia, inhaled prostacyclin via HFNC at 30-50 L/min was reported to decrease the mean pulmonary arterial pressure and/or to improve oxygenation. ${ }^{34-36}$ In all, there is increasing evidence that supports the use of transnasal pulmonary aerosol delivery. In contrast, the evidence is against administering an aerosol by a mask or mouthpiece with concurrent HFNC because it reduced the inhaled dose to as low as $10 \%$ of the inhaled dose achieved with transnasal aerosol delivery. ${ }^{37}$ Consequently, the use of a nebulizer with a mask or mouthpiece concurrent with HFNC should be avoided. ${ }^{23}$ In addition, discontinuing $\mathrm{HFNC}$ to use a conventional nebulizer interrupts HFNC therapy with no advantage in aerosol delivery efficiency. ${ }^{23}$ More efforts are warranted to close the knowledge gap.

The inhaled dose has been reported to increase as the HFNC gas flow decreases ${ }^{23,36,38,39}$; thus, flow reduction may be recommended during aerosol delivery via HFNC. $^{23,40}$ In the survey, only $30 \%$ of the participants reported decreasing the flow during aerosol delivery via HFNC. Moreover, the vibrating mesh nebulizer was the most frequently used nebulizer during transnasal aerosol delivery, especially in North America, $75 \%$ of clinicians used a vibrating mesh nebulizer to deliver aerosol via HFNC, which was in agreement with the survey results among pediatric respiratory therapists (77\%). ${ }^{20}$ This finding might be due to the higher efficiency of transnasal aerosol delivery with a vibrating mesh nebulizer than a small-volume jet nebulizer ${ }^{41}$ and that a vibrating mesh nebulizer does not add a secondary gas flow with a different $\mathrm{F}_{\mathrm{IO}_{2}}$, temperature, and humidity into the HFNC circuit. ${ }^{23}$

\section{Limitations}

Even though there were some incomplete responses, the overall results of the survey may be considered reliable due to the following: (1) the participants were seasoned clinicians, with a mean ICU working experience of 10 years, and $>50 \%$ of them worked in leadership, and (2) all the participants were required to have used HFNC to treat patients within 30 days, which ensured that the answers reflected the current practice in their institutions. Not all the respondents who clicked on the survey and fulfilled the eligibility criteria completed the entire survey, especially the section of aerosol delivery via HFNC that only two-thirds of respondents completed. It might be because the survey was lengthy or that aerosol delivery via HFNC was not in their clinical practice. As such, the actual use of transnasal aerosol delivery might be lower than the reported rate.

\section{Conclusions}

HFNC is broadly used, particularly in patients who are hypoxemic. However, HFNC was mainly used empirically and the detailed information about HFNC utilization, including flow settings and adjustments, varied widely. Consensus or guidelines on HFNC utilization may be warranted. One fourth of the participants delivered aerosol via HFNC, whereas the remaining participants provided aerosol therapy either via nebulizer with a mask or mouthpiece on top of the nasal cannula or discontinued HFNC to use conventional nebulization. The current practice represents a mismatch with available evidence, which suggests the need for efforts to fill the knowledge gaps.

\section{ACKNOWLEDGMENTS}

We thank all the anonymous participants for filling out the survey. We also thank the American Association for Respiratory Care, European Society of Intensive Care Medicine, Chinese Respiratory Disease Society, and Taiwan Respiratory Care Society for endorsing this survey and posting the questionnaire survey on their public media. 


\section{Worldwide Clinical Practice of HFNC}

\section{REFERENCES}

1. Rochwerg B, Einav S, Chaudhuri D, Mancebo J, Mauri T, Helviz Y, et al. The role for high flow nasal cannula as a respiratory support strategy in adults: a clinical practice guideline. Intensive Care Med 2020;46(12):2226-2237.

2. Li J, Jing G, Scott JB. Year in review 2019: high-flow nasal cannula oxygen therapy for adult subjects. Respir Care 2020;65(4):545-557.

3. Rochwerg B, Granton D, Wang DX, Helviz Y, Einav S, Frat JP, et al. High flow nasal cannula compared with conventional oxygen therapy for acute hypoxemic respiratory failure: a systematic review and metaanalysis. Intensive Care Med 2019;45(5):563-572.

4. Granton D, Chaudhuri D, Wang D, Einav S, Helviz Y, Mauri T, et al. High-flow nasal cannula compared with conventional oxygen therapy or noninvasive ventilation immediately postextubation: a systematic review and meta-analysis. Crit Care Med 2020;48(11):e1129-e1136.

5. Chaudhuri D, Granton D, Wang DX, Burns KEA, Helviz Y, Einav S, et al. High-flow nasal cannula in the immediate postoperative period: a systematic review and meta-analysis. Chest 2020;158(5):1934-1946.

6. Leone M, Einav S, Chiumello D, Constantin JM, De Robertis E, De Abreu MG, et al, Guideline contributors. Noninvasive respiratory support in the hypoxaemic peri-operative/periprocedural patient: a joint ESA/ESICM guideline. Intensive Care Med 2020;46(4):697-713.

7. Sang L, Nong L, Zheng Y, Xu Y, Chen S, Zhang Y, et al. Effect of high-flow nasal cannula versus conventional oxygen therapy and noninvasive ventilation for preventing reintubation: a Bayesian network meta-analysis and systematic review. J Thorac Dis 2020;12(7):37253736.

8. Frat JP, Thille AW, Mercat A, Girault C, Ragot S, Perbet S, et al, REVA Network. High-flow oxygen through nasal cannula in acute hypoxemic respiratory failure. N Engl J Med 2015;372(23):21852196.

9. Fong KM, Au SY, Ng GWY. Preoxygenation before intubation in adult patients with acute hypoxemic respiratory failure: a network meta-analysis of randomized trials. Crit Care 2019;23(1):319.

10. Cortegiani A, Crimi C, Sanfilippo F, Noto A, Di Falco D, Grasselli G, et al. High flow nasal therapy in immunocompromised patients with acute respiratory failure: a systematic review and meta-analysis. J Crit Care 2019;50:250-256.

11. Fraser JF, Spooner AJ, Dunster KR, Anstey CM, Corley A. Nasal high flow oxygen therapy in patients with COPD reduces respiratory rate and tissue carbon dioxide while increasing tidal and end-expiratory lung volumes: a randomised crossover trial. Thorax 2016;71(8):759761.

12. Li XY, Tang X, Wang R, Yuan X, Zhao Y, Wang L, et al. High-flow nasal cannula for chronic obstructive pulmonary disease with acute compensated hypercapnic respiratory failure: a randomized, controlled trial. Int J Chron Obstruct Pulmon Dis 2020;15:3051-3061.

13. Jing G, Li J, Hao D, Wang T, Sun Y, Tian H, et al. Comparison of high flow nasal cannula with noninvasive ventilation in chronic obstructive pulmonary disease patients with hypercapnia in preventing postextubation respiratory failure: a pilot randomized controlled trial. Res Nurs Health 2019;42(3):217-225.

14. Fu C, Liu X, Zhu Q, Wu X, Hao S, Xie L, et al. Efficiency of highflow nasal cannula on pulmonary rehabilitation in COPD patients: a meta-analysis. Biomed Res Int 2020;2020:7097243.

15. Elshof J, Duiverman ML. Clinical evidence of nasal high-flow therapy in chronic obstructive pulmonary disease patients. Respiration 2020;99 (2):140-153.

16. Mauri T, Alban L, Turrini C, Cambiaghi B, Carlesso E, Taccone P, et al. Optimum support by high-flow nasal cannula in acute hypoxemic respiratory failure: effects of increasing flow rates. Intensive Care Med 2017;43(10):1453-1463.

17. Mauri T, Turrini C, Eronia N, Grasselli G, Volta CA, Bellani G, Pesenti A. Physiologic effects of high-flow nasal cannula in acute hypoxemic respiratory failure. Am J Respir Crit Care Med 2017;195 (9):1207-1215.

18. Biselli P, Fricke K, Grote L, Braun AT, Kirkness J, Smith P, et al. Reductions in dead space ventilation with nasal high flow depend on physiological dead space volume: metabolic hood measurements during sleep in patients with COPD and controls. Eur Respir J 2018;51 (5): 1702251.

19. Zochios V, Collier T, Blaudszun G, Butchart A, Earwaker M, Jones N, Klein AA. The effect of high-flow nasal oxygen on hospital length of stay in cardiac surgical patients at high risk for respiratory complications: a randomised controlled trial. Anaesthesia 2018;73(12):14781488.

20. Miller AG, Gentle MA, Tyler LM, Napolitano N. High-flow nasal cannula in pediatric patients: a survey of clinical practice. Respir Care 2018;63(7):894-899.

21. Eklund WM, Scott PA. High-flow nasal cannula practice patterns reported by neonatologists and neonatal nurse practitioners in the United States. Adv Neonatal Care 2018;18(5):400-412.

22. Besnier E, Hobeika S, NSeir S, Lambiotte F, Du Cheyron D, Sauneuf $\mathrm{B}$, et al, BoReal study group. High-flow nasal cannula therapy: clinical practice in intensive care units. Ann Intensive Care 2019;9(1):98.

23. Li J, Fink JB, MacLoughlin R, Dhand R. A narrative review on transnasal pulmonary aerosol delivery. Crit Care 2020;24(1):506.

24. Shen Y, Cai G, Yan J. Effect of high flow nasal cannula therapy may be modified by $\mathrm{P}_{\mathrm{aO} 2} / \mathrm{F}_{\mathrm{IO} 2}$ ratio in acute hypoxemic respiratory failure. Intensive Care Med 2019;45(8):1169-1170.

25. Nielsen KR, Ellington LE, Gray AJ, Stanberry LI, Smith LS, DiBlasi RM. Effect of high-flow nasal cannula on expiratory pressure and ventilation in infant, pediatric, and adult models. Respir Care 2018;63 (2): 147-157.

26. Ritchie JE, Williams AB, Gerard C, Hockey H. Evaluation of a humidified nasal high-flow oxygen system, using oxygraphy, capnography and measurement of upper airway pressures. Anaesth Intensive Care 2011;39(6): 1103-1110.

27. Ari A, Fink JB. Effective bronchodilator resuscitation of children in the emergency room: device or interface? Respir Care 2011;56(6):882-885.

28. Morgan SE, Mosakowski S, Solano P, Hall JB, Tung A. High-flow nasal cannula and aerosolized $\beta$ agonists for rescue therapy in children with bronchiolitis: a case series. Respir Care 2015;60(9):e161e165.

29. Valencia-Ramos J, Mirás A, Cilla A, Ochoa C, Arnaez J. Incorporating a nebulizer system into high-flow nasal cannula improves comfort in infants with bronchiolitis. Respir Care 2018;63(7):886-893.

30. Baudin F, Buisson A, Vanel B, Massenavette B, Pouyau R, Javouhey E. Nasal high flow in management of children with status asthmaticus: a retrospective observational study. Ann Intensive Care 2017;7(1):55.

31. Bráunlich J, Wirtz H. Oral versus nasal high-flow bronchodilator inhalation in chronic obstructive pulmonary disease. J Aerosol Med Pulm Drug Deliv 2018;31(4):248-254.

32. Reminiac F, Vecellio L, Bodet-Contentin L, Gissot V, Le Pennec D, Salmon Gandonniere C, et al. Nasal high-flow bronchodilator nebulization: a randomized cross-over study. Ann Intensive Care 2018;8 (1): 128 .

33. Li J, Zhao M, Hadeer M, Luo J, Fink JB. Dose response to transnasal pulmonary administration of bronchodilator aerosols via nasal highflow therapy in adults with stable chronic obstructive pulmonary disease and asthma. Respiration 2019;98(5):401-409.

34. Ammar MA, Sasidhar M, Lam SW. Inhaled epoprostenol through noninvasive routes of ventilator support systems. Ann Pharmacother 2018;52(12):1173-1181.

35. Li J, Harnois LJ, Markos B, Roberts KM, Homoud SA, Liu J, et al. Epoprostenol delivered via high flow nasal cannula for ICU subjects with severe hypoxemia comorbid with pulmonary hypertension or right heart dysfunction. Pharmaceutics 2019;11(6):281. 


\section{Worldwide Clinical Practice of HFNC}

36. Li J, Gurnani PK, Roberts KM, Fink JB, Vines D. The clinical impact of flow titration on epoprostenol delivery via high flow nasal cannula for ICU patients with pulmonary hypertension or right ventricular dysfunction: a retrospective cohort comparison study. J Clin Med 2020;9(2):464.

37. Bennett G, Joyce M, Fernández EF, MacLoughlin R. Comparison of aerosol delivery across combinations of drug delivery interfaces with and without concurrent high-flow nasal therapy. Intensive Care Med Exp 2019;7(1):20.

38. Alcoforado L, Ari A, de Melo Barcelar J, Brandão SCS, Fink JB, de Andrade AD. Impact of gas flow and humidity on trans-nasal aerosol deposition via nasal cannula in adults: a randomized cross-over study. Pharmaceutics 2019;11(7):320.
39. Li J, Gong L, Fink JB. The ratio of nasal cannula gas flow to patient inspiratory flow on trans-nasal pulmonary aerosol delivery for adults: an in vitro study. Pharmaceutics 2019;11(5):225.

40. Ministry of Health in New South Wales Australia. Humidified high flow nasal cannula oxygen guideline for metropolitan paediatric wards and EDs. 2020. Available at: http://www1.health.nsw.gov.au/pds/ ActivePDSDocuments/GL2016_004.pdf. Accessed November 11, 2020.

41. Dugernier J, Hesse M, Jumetz T, Bialais E, Roeseler J, Depoortere $\mathrm{V}$, et al. Aerosol delivery with two nebulizers through high-flow nasal cannula: a randomized cross-over single-photon emission computed tomography study. J Aerosol Med Pulm Drug Deliv 2017;30(5):349-358 